\title{
Neurocircuitry: A Window into the Networks Underlying Neuropsychiatric Disease
}

U nderstanding the neurocircuitry that underlies normal and adaptive behaviors is a foundation for elucidating the pathology and pathophysiology of psychiatric diseases. The rich history of anatomical observations forms the backbone for what we now consider to be the key structures and pathways associated with aberrant behaviors and psychiatric disorders. Given the focus of psychiatry on the interface of motivation, emotion, and cognition, psychiatric neuroscience has progressively highlighted brain networks that subserve these functional domains and their interactions. The convergence of findings from the variety of techniques in animal studies to human postmortem, neurosurgical, and brain imaging methods have led to major advances that constitute the groundwork for the neurocircuitry pertinent to psychiatric diseases.

The goal of this volume is to outline the circuitries that are thought to underlie psychiatric disorders, with a particular focus on recent work in the field, as it relates to normal and abnormal behaviors. One of the first key anatomical contributions was the development of the idea that specific connected brain regions control emotion (the limbic system). Although the concept of the limbic system as originally conceptualized may be outdated, a constellation of brain structures appears to be at the core of most neuropsychiatric disorders. The key structures include the prefrontal cortex, the striatum, the hippocampus, the amygdala (including the extended amygdala), and the ascending monoaminergic modulatory transmitter systems, particularly the midbrain dopamine system. Clearly, this is not an exhaustive list, but one that is essential.

Each chapter in the volume highlights one or more of these linked structures within the complex neural networks that underlie aberrant behaviors relevant to psychiatry (Figure 1). Several chapters cover anatomical constructs in detail, whereas others refer more broadly to 'circuits'. Given that many of the structures are repeatedly in focus throughout the volume, the combination of more anatomically based reviews and papers emphasizing behavior and disease gives rise to a volume that contains the necessary anatomical details, without detracting from our overarching goal of understanding the relationship between structure and function.

The first part of the volume focuses on the neurocircuitry of normal functions and development, and the second part on neuropsychiatric disorders and their treatments. Chapters 1 and 2 discuss the circuitry of the reward system:
Haber and Knutson review the pertinent pathways at the macro level with an emphasis on relating primate anatomy of the prefrontal-basal ganglia circuit with imaging studies in humans; Sesack and Grace address the microcircuitry in the rat, with a focus on how convergence of hippocampal, amygdala, and prefrontal cortical inputs to the nucleus accumbens, modulated by dopamine, affects goal-directed behavior. The next three chapters spotlight normal cognitive functions: goal-directed behavior, executive function, and memory. Balleine and O'Dougherty compare rodent and human studies and the role of the cortico-striatal circuitry in learning and in the motivational control of actions. Leh et al summarize the neurobiological underpinnings of executive function (focused on frontal cortex) based on integrating neuroimaging with pharmacological and transcranial magnetic stimulation methods and recent insights from dopamine loss in Parkinson's disease. Dickerson and Eichenbaum review the role of the medial temporal lobe in cognition along with the neurobiological basis of episodic memory and the disorders associated with it. Chapters 6 and 7 discuss the neurocircuitry of anxiety and emotion regulation. Davis et al show the importance of the extended amygdala in models of fear and anxiety, focusing on a rodent model. Hartley and Phelps review both animal models and human studies of different methods that regulate emotion, focusing on the central position of the amygdala and its relationship to the prefrontal cortex, hippocampus, and the striatum. In chapter 8, Tau and Peterson review the normal development of cortical circuits, providing the necessary template to identify the developmental origins of neuropsychiatric illnesses.

Chapters 9-14 focus on specific psychiatric disorders. In chapter 9, Shin and Liberzon continue the discussion of anxiety and emotion regulation by reviewing the anatomy of anxiety disorders with an amygdalo-cortical focus. In chapter 10, Price and Drevets review the circuits that link structures associated with mood disorders, with a particular focus on the ventral medial prefrontal cortex, and its relationship to subcortical structures. In chapter 11, Koob and Volkow then review the neurocircuitry that is engaged at each of the three stages of the addiction cycle: the ventral striatum and ventral tegmental area; the extended amygdala; and a more distributed network that includes the prefrontal cortex and amgydala. Chapters 12 and 13 address the landscape of neurocircuitry pertaining to schizophrenia 


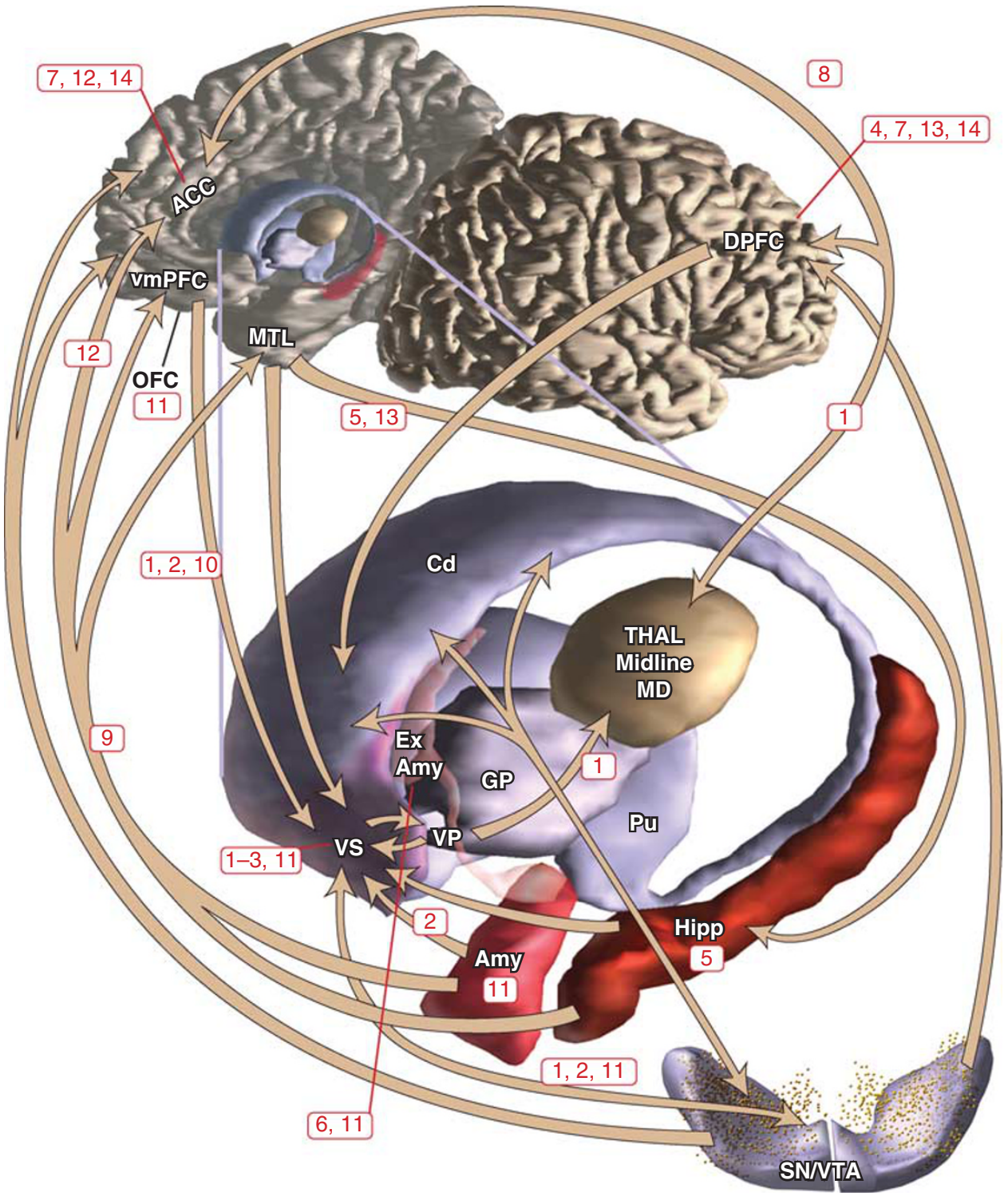

Figure 1. Schematic illustrating some key structures and pathways involved in neuropsychiatric disorders. Arrows illustrate projections; numbers refer to chapters in which those structures or pathways are discussed. Amy, amygdala; ACC, anterior cingulate cortex; Cd, caudate nucleus; DPFC, dorsal prefrontal cortex; GP, globus pallidus; Ex Amy, extended amygdala; Hipp, hippocampus; MD, medial dorsal nucleus of the thalamus; MTL, medial temporal lobe; OFC, orbital frontal cortex; Pu, Putamen; SN, substantia nigra; yellow dots, dopamine neurons; Thal, thalamus; vmPFC, ventral medial prefrontal cortex; VP, ventral pallidum; VS, ventral striatum; VTA, ventral tegmental area.

and psychosis. Benes' review builds on an extensive literature from neuropathology, concentrating on the amygdalo-cortical circuitry. Eisenberg and Berman follow this with a review of the circuitry of executive function in schizophrenia and the role of genetic polymorphisms, focusing primarily on the prefrontal cortex, but taking into account the importance of the inferior parietal lobe, medial temporal cortex and pathways through the basal ganglia and the thalamus. In chapter 14, Bush reviews the synergistic contributions of cognitive neuroscience and neuroimaging to contemporary models of attention-deficit disorder. In particular, he outlines the cingulo-frontal-parietal attentional network, the 'default mode' resting-state network, and reward/feedback-based processing circuitry.
The final two articles illustrate how knowledge about neurocircuitry in animals and human neuroimaging studies can be leveraged in the service of emerging therapies. In chapter 15, George and Aston-Jones review noninvasive neurostimulation therapies, such as transcranial magnetic stimulation for major depression. In chapter 16, Greenberg et al review neurosurgical treatments, including deep brain stimulation as invasive interventions, taking as an example, obsessive-compulsive disorder.

Thus, this volume progresses from the basic circuitry of normal behaviors to those underlying psychiatric disorders and their treatment. As a field, we can reflect on a history of iterative refinement in our appreciation of the brain's complex architecture, at the micro, macro, and systems 
levels. Moreover, knowledge regarding the interplay between normal anatomy and physiology paves the way for discovering the basis of psychiatric diseases. This in turn opens new vistas for developing better means for diagnosis and treatment strategies, and ultimately prevention or cure. The volume aptly captures the momentum and acceleration this field is experiencing. By mapping neurocircuitry, we provide a window into the networks underlying neuropsychiatric disease - a critical pursuit towards delivering on the promise of translational neuroscience.

We thank the Hot Topics' editor, Dr Kathryn Cunningham, who provided a compendium of outstanding perspective articles highlighting some of the most exciting findings and scientific viewpoints from the last year. We also thank the ACNP, especially Diane Drexler, and Katie Ris-Vicari at Nature Publishing, for their untiring devotion to this project and its demanding timeline.

\section{DISCLOSURE}

Dr Haber has received honoraria fees from Lilly and consultation fees from Medtronic. Dr Rauch has received honoraria and/or consultation fees from Neurogen, Sepracor, Novartis, and Medtronic. He has also participated in research funded by Medtronic, Cyberonics, Cephalon, and Northstar. A comprehensive disclosure for SLR is supplied as Supplemental Material online.

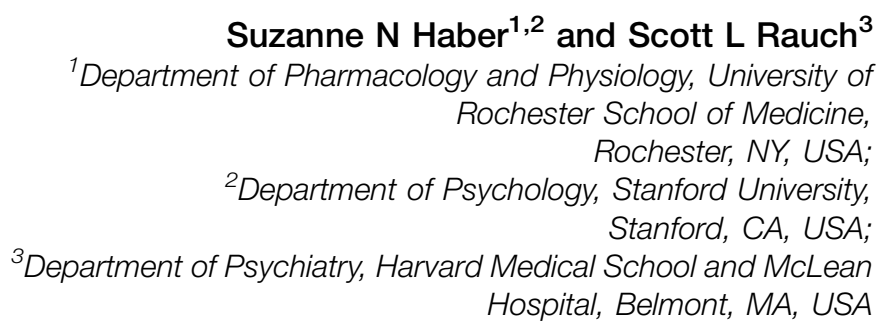

Supplementary Information accompanies the paper on the Neuropsychopharmacology website (http://www.nature.com/npp) 